\title{
MOTION OF A CHARGED PARTICLE IN A RANDOMLY VARYING MAGNETIC FIELD
}

\author{
A. Ziya AKCASU \\ Department of Nuclear Engineering, University of Michigan, Ann Arbor, MI 48109, USA \\ and \\ Boualem HAMMOUDA \\ Research Reactor Facility, University of Missouri, Columbia, MO 65211, USA
}

Received 13 November 1984

The motion of a charged particle in a randomly varying uniform magnetic field is investigated. Stability of the mean position and mean square displacement are determined rigorously in the case of random binary variations (telegraph signal). It is found that the mean position can be stable if the fluctuating part of the magnetic field is large enough compared to the uniform background. The stability regions are obtained in the parameter plane. It is also found that the mean square displacement can be unstable even in the region where the mean is stable. After an initial diffusive motion (with a calculable diffusion coefficient), the charged particle either acquires wild oscillations in its trajectory (stable mean, unstable mean square) or quickly leaves the region where it started (unstable mean).

\section{Introduction}

The motion of charged particles in deterministically time-dependent uniform magnetic fields was studied by Chandrasekhar ${ }^{1}$ ) in the late fifties in order to discuss the validity of the adiabatic invariants of charged particles in timevarying magnetic fields. He constructed an approximate series solution of the nonrelativistic equation of motion of a particle with mass $m$ and charge $q$ :

$$
m \mathrm{~d} v / \mathrm{d} t=q[E+(1 / c) v \times B]
$$

in which the magnetic field was assumed to be uniform in space but a function of time as

$$
B(t)=e_{z} B(t),
$$

where $e_{z}$ is the constant unit vector in the direction of $B$, taken to be the $z$-axis. 
In the absence of net space charges, the electric field $E$ in (1) satisfies

$$
\boldsymbol{\nabla} \cdot \boldsymbol{E}=0, \quad \boldsymbol{\nabla} \times \boldsymbol{E}=(1 / c) \partial \boldsymbol{B} / \partial t
$$

The cylindrically symmetric solution of eq. (3) is

$$
E(r, t)=(1 / 2 c) r \times e_{z} \dot{B}(t)
$$

where $\dot{B}(t)$ denotes the time derivative. Substitution of (4) into (1) yields

$$
\dot{v}=\omega(t) v \times e_{z}+(1 / 2) \dot{\omega}(t) r \times e_{z}
$$

where $\omega(t)=q B(t) / m c$ is the instantaneous Larmor frequency. Since the motion of the particle along the field lines is of no particular interest, we set $\boldsymbol{e}_{z} \cdot \boldsymbol{v}=0$, and investigate the motion of the particle on the $x-y$ plane, i.e. perpendicular to the field lines by our choice. By introducing the complex variables $z=x+\mathrm{i} y$ and $\dot{z}=v_{x}+\mathrm{i} v_{y}$, the vector equation (5) can be transformed into a complex differential equation

$$
\mathrm{d}^{2} z / \mathrm{d} t^{2}+\mathrm{i} \omega(t) \dot{z}+(\mathrm{i} / 2) \dot{\omega}(t) z=0
$$

Using this form of the equation of motion, Chandrasekhar ${ }^{\prime}$ ) calculated exactly the change in the magnetic moment of a charged particle following a step change in the strength of the magnetic field.

In this paper, we extend Chandrasekhar's work, and investigate the motion of a charged particle using eq. (6) when the magnetic field $B(t)$ is subject to random fluctuations. In this case $\omega(t)$ in eq. (6) is assumed to be a particular realization of a known stationary random process, and the mean and mean square values of the solution $z(t)$ of the resulting stochastic differential equation are sought. We calculate in particular the mean square displacement of the particle as function of time, and show that the particle executes a diffusion motion in the vicinity of the origin which is chosen to be the center of symmetry assumed in solving Maxwell's equations (3) to obtain the induced electric field. We determine the diffusion coefficient in terms of the power spectrum of the magnetic field fluctuations. Away from the origin, the induced electric field becomes dominant, and the mean square distance increases exponentially in time in contrast to the linear increase near the origin.

Since the stochastic differential equation (6) is linear, the subtle difficulties inherent in most nonlinear stochastic equations elucidated by Van Kampen ${ }^{2,3}$ ) do not arise in our discussions. 


\section{Behavior near the origin}

We first consider the motion of the particle near the center of symmetry where the effect of the induced electric field represented by the last term in eq. (6) is expected to be small. We let

$$
\omega(t)=\omega_{0}[1+n(t)]
$$

where $n(t)$ is the fluctuating part of the Larmor frequency with zero mean. We set $\omega_{0} t \rightarrow t$, i.e. the unit of time as the Larmor period. The stochastic differential equation (6) then becomes

$$
\mathrm{d}^{2} z / \mathrm{d} t^{2}+\mathrm{i}[1+n(t)] \dot{z}=0
$$

which can easily be solved as

$$
z(t)=z_{0}+\dot{z}_{0} \int_{0}^{t} \mathrm{~d} u \mathrm{e}^{-\mathrm{i} u} \mathrm{e}^{-\mathrm{i} \theta(u)}
$$

where $z_{0}$ and $\dot{z}_{0}$ are the initial position and velocity of the charged particle in complex notation, and

$$
\theta(t) \equiv \int_{0}^{t} \mathrm{~d} u n(u)
$$

The mean position, mean square displacement, and velocity autocorrelation function of the particle are obtained from (8) as:

$$
\begin{aligned}
& \langle z(t)\rangle=z_{0}+\dot{z}_{0} \int_{0}^{t} \mathrm{~d} u \mathrm{e}^{-\mathrm{i} u}\left\langle\mathrm{e}^{-\mathrm{i} \theta(u)}\right\rangle, \\
& \left\langle|\Delta z(t)|^{2}\right\rangle=2\left|\dot{z}_{0}\right|^{2} \int_{0}^{t} \mathrm{~d} u(t-u) \operatorname{Re}\left[\left\langle\mathrm{e}^{-\mathrm{i} u} \mathrm{e}^{-\mathrm{i} \theta(u)}\right\rangle\right],
\end{aligned}
$$

and

$$
\phi(t)=\left|\dot{z}_{0}\right|^{2} \operatorname{Re}\left[\left\langle\mathrm{c}^{-\mathrm{i} t} \mathrm{e}^{-\mathrm{i} \theta(t)}\right\rangle\right]
$$


In reducing the double integration to a single integration in the derivation of (11), we assumed the stationarity of $n(t)$ which implies that $\langle\exp [\mathrm{i} \theta(u)-\mathrm{i} \theta(v)]\rangle$ is a function of $(u-v)$ only. It is observed that the calculation of the mean, mean square displacement, and velocity autocorrelation function involve the characteristic function $\langle\exp [\mathrm{i} \theta(t)]\rangle$ of the random variable $\theta(t)$. Both eqs. (11) and (12) can be used to calculate the diffusion coefficient from

$$
\lim _{t \rightarrow \infty}\left\langle|\Delta z(t)|^{2}\right\rangle=4 D t
$$

i.e.

$$
D=(1 / 2) \int_{0}^{\infty} \mathrm{d} u \phi(u)
$$

or assuming that the initial velocity distribution is $\left\langle\left|\dot{z}_{0}\right|^{2}\right\rangle=2 k_{\mathrm{B}} T / m$ :

$$
D=\left(k_{\mathrm{B}} T / m\right) \operatorname{Re}\left[\int_{0} \mathrm{~d} u \mathrm{e}^{-\mathrm{i} u}\left\langle\mathrm{e}^{-\mathrm{i} \theta(u)}\right\rangle\right] .
$$

In order to illustrate the application of these results, we consider two examples in which $n(t)$ represents a Gaussian process and a binary telegraph signal.

\section{Gaussian process}

When $n(t)$ is taken to be a Gaussian process with zcro mean and a correlation function $\Phi(\tau)=\langle n(0) n(\tau)\rangle$, the characteristic function of $\theta(t)$ is obtained as

$$
\langle\exp [-\mathrm{i} \theta(t)]\rangle=\exp \left[-\int_{0}^{t} \mathrm{~d} s(t-s) \Phi(s)\right] .
$$

Once $\Phi(t)$ is specified, the above statistical quantities can be calculated numerically. To obtain some physical insight into the motion of the particle analytically, we consider a white Gaussian process such that $\Phi(\tau)=G_{0} \delta(\tau)$ where $G_{0}$ is the constant value of the power spectral density of the white noise process:

$$
\langle z(t)\rangle=z_{0}+\dot{z}_{0}\left[1-\mathrm{e}^{-\left(\mathrm{i}+G_{0} / 2\right) t}\right] /\left[\mathrm{i}+G_{0} / 2\right]
$$


and

$$
D=\left(k_{\mathrm{B}} T / m\right)\left(G_{0} / 2\right) /\left(1+G_{0}^{2} / 4\right) .
$$

Eq. (15) indicates that the mean position of the particle after long times is shifted to

$$
\langle z(\infty)\rangle=z_{0}+\dot{z}_{0} /\left(\mathrm{i}+G_{0} / 2\right) .
$$

As an example, we assume that the particle is initially located at the origin and has a velocity $v_{0}$ along the $y$-axis, i.e. $z_{0}=0$ and $\dot{z}_{0}=\mathrm{i} v_{0}$. The coordinates of the final position follow from (17) as

$$
\langle x(\infty)\rangle=v_{0} /\left(1+G_{0}^{2} / 4\right), \quad\langle y(\infty)\rangle=v_{0}\left(G_{0} / 2\right) /\left(1+G_{0}^{2} / 4\right) .
$$

In the absence of fluctuations, viz. $G_{0}=0,\langle z(t)\rangle$ displays the usual gyration of the charged particle in a constant uniform magnetic field. The asymptotic mean position coincides with the initial position of the particle when its initial velocity distribution is isotropic.

The fluctuations in the magnetic field produce a diffusional motion exactly the same way as collisions would. The diffusion coefficient has a maximum when $G_{0}=2$ or in real time $G_{0}=2 \omega_{0}$. The physical interpretation of this condition is not clear to us.

\section{Binary telegraph signal}

In this case, $n(t)$ is assumed to be a random process whose member functions are defined as follows: Each function $n(t)$ takes only two values $+\eta$ or $-\eta$ and makes independent random jumps between these two values. The probability of having $m$ sign reversals in a time interval $T$ is given by a Poisson distribution:

$$
P(m, T)=\mathrm{e}^{-\lambda T}(\lambda T)^{m} / m !,
$$

where $\lambda$ is the mean reversal rate. In order to calculate the characteristic function $\langle\exp [-\mathrm{i} \theta(t)]\rangle$, we express the probability density $P(\theta, t)$ as

$$
P(\theta, t)=\sum_{n} P(\theta, n, t),
$$

where $P(\theta, n, t)$ is the joint probability density for the joint process $\{\theta(t), n(t)\}$, 
and summation over $n$ implies $n=+\eta$ and $n=-\eta$. The reason for introducing $P(\theta, n, t)$ is that the joint process $\{\theta(t), n(t)\}$ is a Markov process and thus its joint probability density satisfies a Chapman-Kolmogorov relation:

$$
P(\theta, n, t+\Delta t)=\int \mathrm{d} \theta^{\prime} \sum_{n^{\prime}} P\left(\theta, n, t+\Delta t \mid \theta^{\prime}, n^{\prime}, t\right) P\left(\theta^{\prime}, n^{\prime}, t\right) .
$$

The transition probability can be written explicitly in this problem as

$$
\begin{aligned}
P\left(\theta, n, t+\Delta t \mid \theta^{\prime}, n^{\prime}, t\right)= & (1-\lambda \Delta t) \delta\left[\theta-\theta^{\prime}-\delta \theta\left(n, \theta^{\prime}\right)\right] \delta\left(n-n^{\prime}\right) \\
& +\lambda \Delta t \delta\left(\theta-\theta^{\prime}\right) \delta\left(n+n^{\prime}\right)+\mathcal{O}\left(\Delta t^{2}\right) .
\end{aligned}
$$

The first term represents a transition in $\Delta t$ in which no sign reversal occurs, and hence $n=n^{\prime}$. The probability of this transition mode is $(1-\lambda \Delta t)$. The Dirac delta function is the conditional probability that the variable $\theta(t)$ will change from $\theta^{\prime}$ to $\theta=\theta^{\prime}+\delta \theta\left(n, \theta^{\prime}\right)$ during this transition. The second term in eq. (21) represents a transition with probability $\lambda \Delta t$ in which a sign reversal occurs so that $n=-n^{\prime}$. Since this term is already proportional to $\Delta t$, the change in $\theta(t)$ during $\Delta t$ leads to terms of higher order in $\Delta t$ and thus can be ignored. Transitions involving more than one sign reversal are also of higher order in $\Delta t$, and hence ignored.

Substituting eq. (21) into (20), expanding the delta function in the first term of eq. (21) as

$$
\delta\left[\theta-\theta^{\prime}-\delta \theta(n, \theta)\right] \simeq \delta\left(\theta-\theta^{\prime}\right)-\delta \theta(n, \theta)\left(\partial \delta\left(\theta-\theta^{\prime}\right) / \partial \theta\right)
$$

and defining

$$
\begin{aligned}
& P_{1}(\theta, t) \equiv P(\theta,+\eta, t), \\
& P_{2}(\theta, t) \equiv P(\theta,-\eta, t),
\end{aligned}
$$

we obtain

$$
\begin{aligned}
& \partial P_{1} / \partial t=-(\partial / \partial \theta)\left[P_{1} \delta \theta^{+}(\theta) / \Delta t\right]+\lambda\left(P_{2}-P_{1}\right) \\
& \partial P_{2} / \partial t=-(\partial / \partial \theta)\left[P_{2} \delta \theta^{-}(\theta) / \Delta t\right]+\lambda\left(P_{1}-P_{2}\right) .
\end{aligned}
$$

The increment $\delta \theta^{ \pm}(\theta)$ in $\theta(t)$ during $\Delta t$ is to be calculated from the differential equations governing the time evolution of $\theta(t)$, in general. In the present application, it is given by 


$$
\delta \theta^{ \pm}(\theta)= \pm \eta \Delta t
$$

so that eqs. (22) become

$$
\begin{aligned}
& \partial P_{1} / \partial t=-\eta \partial P_{1} / \partial \theta+\lambda\left(P_{2}-P_{1}\right), \\
& \partial P_{2} / \partial t=\eta \partial P_{2} / \partial \theta+\lambda\left(P_{1}-P_{2}\right) .
\end{aligned}
$$

The desired probability density $P(\theta, t)$ is given by $P(\theta, t)=P_{1}(\theta, t)+P_{2}(\theta, t)$ according to eq. (19). Its time evolution is obtained from eqs. (23) as

$$
\partial^{2} P / \partial t^{2}+2 \lambda \partial P / \partial t=\eta^{2} \partial^{2} P / \partial \theta^{2} .
$$

Since we are mainly interested in the characteristic function $C(k, t) \equiv$ $\langle\exp [-\mathrm{i} k \theta(t)]\rangle$ with $k=1$, we Fourier transform eq. (24) to obtain our equation for $C(k, t)$ directly:

$$
\partial^{2} C / \partial t^{2}+2 \lambda \partial C / \partial t=-k^{2} \eta^{2} C
$$

The initial conditions for $C(k, t)$ are $C(k, 0)=1$ and $\dot{C}(k, 0)=0$. They follow from $P_{1}(\theta, 0)=\delta(\theta) / 2$ and $P_{2}(\theta, 0)=\delta(\theta) / 2$, where we assume that $n(0)= \pm \eta$ with equal probability. These imply $P(\theta, 0)=\delta(\theta)$ and $P_{1}(\theta, 0)-P_{2}(\theta, 0)=0$. The former leads to $C(k, 0)=1$. The latter leads, with $\dot{P}(\theta, t)=$ $-\eta \partial\left(P_{1}-P_{2}\right) / \partial \theta$ or $\dot{C}(k, t)=-\mathrm{i} \eta k\left[\bar{P}_{1}(k, t)-\bar{P}_{2}(k, t)\right]$, to $\dot{C}(k, 0)=0$. Hence the solution of eq. (25) with these initial conditions yields

$$
\left\langle\mathrm{e}^{-\mathrm{i} \theta(t)}\right\rangle=\mathrm{e}^{-\lambda t}[(\lambda / \Omega) \sin (\Omega t)+\cos (\Omega t)],
$$

where $\Omega \equiv\left(\eta^{2}-\lambda^{2}\right)^{1 / 2}$. The mean position $\langle z(t)\rangle$, mean square displacement $\left\langle\left.\Delta z(t)\right|^{2}\right\rangle$, and velocity auto-correlation function $\phi(t)$ are obtained by substituting eq. (26) into eqs. (10), (11) and (12) respectively. The result in eq. (26) is valid for both $\eta>\lambda$ and $\eta<\lambda$. In the latter case, $\Omega$ is replaced by $i \bar{\Omega}$ with $\bar{\Omega}=\left(\lambda^{2}-\eta^{2}\right)^{1 / 2}$. Since $-\lambda+\left(\lambda^{2}-\eta^{2}\right)^{1 / 2}<0,\langle\exp [i \theta(t)]\rangle$ is a decaying function of time in both cases. We present the velocity auto-correlation function explicitly when $\eta>\lambda$ :

$$
\phi(t)=\phi(0) \cos (t) \mathrm{e}^{-\lambda t}[(\lambda / \Omega) \sin (\Omega t)+\cos (\Omega t)],
$$

and when $\eta<\lambda$ :

$$
\phi(t)=\phi(0) \cos (t) \mathrm{e}^{-\lambda t}[(\lambda / \Omega) \sinh (\Omega t)+\cosh (\Omega t)],
$$


where $\Omega=\left|\eta^{2}-\lambda^{2}\right|^{1 / 2}$ in both cases. The diffusion coefficient is obtained using eq. (13a) as

$$
D=\left(k_{\mathrm{B}} T / m\right)\left(\eta^{2} / 2 \lambda\right) /\left[1+\left(\left(\eta^{2}-1\right) / 2 \lambda\right)^{2}\right]
$$

in either case.

The auto-correlation function and power spectral density of a random binary signal process as defined above are, respectively, $\eta^{2} \exp (-2 \lambda|\tau|)$ and $\left(\eta^{2} / \lambda\right) /[1+$ $\left.\left(\eta^{2} / 2 \lambda\right)^{2}\right]$. In the limit of $\lambda \rightarrow \infty$ with $\eta^{2} / \lambda=G_{0}$ where $G_{0}$ is constant, this process approaches to a white noise process with a power spectral density $G_{0}$, and eq. (28) reproduces the diffusion coefficient in eq. (16) calculated with the Gaussian white noise process with the same $G_{0}$. The diffusion coefficient vanishes in the limit of $\lambda \rightarrow 0$ as expected because this limit corresponds to a single step change in the magnetic field rather than a sequence of step changes. An interesting special case is $\eta=1$ which corresponds to binary changes in the magnetic field strength between zero and $2 B_{0}$. In this case, the diffusion coefficient is proportional to $1 / \lambda$ and increases when the mean reversal rate is decreased. This may be expected because the particle moves on a straight line during those time intervals where $B=0$.

\section{Complete solution of eq. (6)}

The stochastic equation (6) governing the random motion of the charged particle in both $\boldsymbol{B}$ and $\boldsymbol{E}$ fields can be treated exactly when $\omega(t)$ is a binary signal process. Following Chandrasekhar's treatment, we substitute in eq. (6):

$$
z(t)=Z(t) \exp \left[-(\mathrm{i} / 2) \int_{0}^{t} \mathrm{~d} t^{\prime} \omega\left(t^{\prime}\right)\right]
$$

to transform it into

$$
\mathrm{d}^{2} Z / \mathrm{d} t^{2}+\left(\omega^{2}(t) / 4\right) Z=0 .
$$

This equation which describes a harmonic oscillator with a random frequency has been investigated by many authors previously ${ }^{47}$ ). In this paper, we follow the approach used by McKenna and Morisson ${ }^{6}$ ) with slight modifications when $\omega(t)$ is the random telegraph binary process.

Eq. (30) has two linearly independent real solutions $X_{1}(t)$ and $X_{2}(t)$ satisfying the following initial conditions:

$$
X_{1}(0)=1, \quad \dot{X}_{1}(0)=0, \quad X_{2}(0)=0, \quad \dot{X}_{2}(0)=1 .
$$


Its general solution can be written as

$$
Z(t)=Z_{0} X_{1}(t)+\dot{Z}_{0} X_{2}(t)
$$

where the constants of integration $Z_{0}$ and $\dot{Z}_{0}$ are related to the initial position and velocity of the charged particle as $Z_{0}=z_{0}$ and $\dot{Z}_{0}=\dot{z}_{0}+i \omega(0) z_{0} / 2$.

As before, we assume that $\omega(t)=\omega_{0}[1+n(t)]$ where $n(t)$ is a binary random process assuming two values $\pm \eta$ randomly. To compress equations, we choose the unit of time such that the dimensionless time is $t=\omega_{0} t_{\text {real }} / 2$. Note that the normalization of time contains the factor $1 / 2$ in this section. Then eq. (29) becomes

$$
z(t)=\left[Z_{0} X_{1}(t)+\dot{Z}_{0} X_{2}(t)\right] \mathrm{e}^{-\mathrm{i} \theta(t)} \mathrm{e}^{-\mathrm{i} t}
$$

where

$$
\theta(t)=\int_{0}^{t} \mathrm{~d} u \eta(u),
$$

and $X_{1}(t)$ and $X_{2}(t)$ now satisfy the dimensionless form of the harmonic oscillator equation

$$
\mathrm{d}^{2} X_{1,2} / \mathrm{d} t^{2}+[1+n(t)]^{2} X_{1,2}=0
$$

with the initial conditions given in eq. (31). We note that $X_{1}(t), X_{2}(t)$ and $\theta(t)$ are three correlated random processes. The calculation of the mean position $\langle z(t)\rangle$ of the charged particle requires the following cross-correlations of these processes:

$$
\begin{aligned}
& g(t) \equiv\left\langle X_{1}(t) \mathrm{e}^{-\mathrm{i} \theta(t)}\right\rangle, \\
& h(t) \equiv\left\langle X_{2}(t) \mathrm{e}^{-\mathrm{i} \theta(t)}\right\rangle .
\end{aligned}
$$

Higher moments of $z(t)$ involve averages like $\left\langle X_{1}^{2}(t)\right\rangle,\left\langle X_{2}^{2}(t)\right\rangle$ and $\left\langle X_{1}(t) X_{2}(t)\right\rangle$, etc. To calculate such averages, one needs the joint probability distribution $P\left(X_{1}, X_{2}, \theta, n, t\right)$. However, the set of variables $\left\{X_{1}, X_{2}, \theta, n\right\}$ does not form a Markov process because $X_{1}(t)$ and $X_{2}(t)$ satisfy a second order differential equation, eq. (35). We augment this set by including $\dot{X}_{1}(t)$ and $\dot{X}_{2}(t)$ to obtain a vector Markov process with components $\{\xi, \theta, n\}$ where

$$
\xi(t) \equiv \operatorname{col}\left[X_{1}, \dot{X}_{1}, X_{2}, \dot{X}_{2}\right] .
$$


The time evolution of $\boldsymbol{\xi}(t)$ follows from eq. (35) as

$$
\dot{\xi}(t)=\mathbf{M}[n(t)] \cdot \xi(t),
$$

where

$$
\mathbf{M}[n]=\left(\begin{array}{cccc}
0 & 1 & 0 & 0 \\
-[1+n]^{2} & 0 & 0 & 0 \\
0 & 0 & 0 & 1 \\
0 & 0 & -[1+n]^{2} & 0
\end{array}\right)
$$

In order to obtain the master equation for the joint distribution $P(\xi, \theta, n, t)$, we again introduce $P_{1}(\xi, \theta, t) \equiv P(\xi, \theta,+\eta, t)$ and $P_{2}(\xi, \theta, t)=P(\xi, \theta,-\eta, t)$. Following the procedure described in the previous section in vectorial form we obtain

$$
\begin{aligned}
& \partial P_{1} / \partial t=-(\partial / \partial \xi) \cdot\left[P_{1} \mathbf{M}^{+} \cdot \xi\right]-(\partial / \partial \theta)\left[\eta P_{1}\right]+\lambda\left[P_{2}-P_{1}\right] \\
& \partial P_{2} / \partial t=-(\partial / \partial \xi) \cdot\left[P_{2} \mathbf{M}^{-} \cdot \xi\right]+(\partial / \partial \theta)\left[\eta P_{2}\right]+\lambda\left[P_{1}-P_{2}\right],
\end{aligned}
$$

where $\mathbf{M}^{ \pm}=\mathbf{M}( \pm \eta)$. We present a derivation of these equations in a more general context in the appendix. A more explicit form of eqs. (39) is

$$
\begin{aligned}
\partial P_{1} / \partial t= & -\left[\dot{X}_{1} \partial P_{1} / \partial X_{1}+\dot{X}_{2} \partial P_{1} / \partial X_{2}\right]+\beta^{+}\left[X_{1} \partial P_{1} / \partial \dot{X}_{1}+X_{2} \partial P_{1} / \partial \dot{X}_{2}\right] \\
& -\eta \partial P_{1} / \partial \theta+\lambda\left(P_{2}-P_{1}\right), \\
\partial P_{2} / \partial \theta= & -\left[\dot{X}_{1} \partial P_{2} / \partial X_{1}+\dot{X}_{2} \partial P_{2} / \partial X_{2}\right]+\beta^{-}\left[X_{1} \partial P_{2} / \partial \dot{X}_{1}+X_{2} \partial P_{2} / \partial \dot{X}_{2}\right] \\
& +\eta \partial P_{2} / \partial \theta+\lambda\left(P_{1}-P_{2}\right),
\end{aligned}
$$

where $\beta^{ \pm}=(1 \pm \eta)^{2}$. Eqs. (40) will serve as the starting equations in calculating the mean and variance of the position $z(t)$ of the particle. The desired probability density $P(\xi, \theta, t)$ is the sum of $P_{1}(\xi, \theta, t)$ and $P_{2}(\xi, \theta, t)$.

The conditional probability densities $P_{1}\left(\xi, \theta, t \mid \xi_{0}, \theta_{0}, n_{0}, 0\right)$ and $P_{2}\left(\xi, \theta, t \mid \xi_{0}, \theta_{0}, n_{0}, 0\right)$ also satisfy these equations with the usual initial conditions.

$$
\begin{aligned}
& P_{1}\left(\xi, \theta, t \mid \xi_{0}, \theta_{0}, n_{0}, 0\right)=\delta\left(\xi-\xi_{0}\right) \delta_{n_{0},+\eta}, \\
& P_{2}\left(\xi, \theta, t \mid \xi_{0}, \theta_{0}, n_{0}, 0\right)=\delta\left(\xi-\xi_{0}\right) \delta\left(\theta-\theta_{0}\right) \delta_{n_{0},-\eta} .
\end{aligned}
$$


We need this somewhat obvious remark later in the calculation of mean position and mean square displacement.

\section{Time evolution of the mean position}

The mean position of the particle follows from eq. (33) as

$$
\langle z(t)\rangle=\left\{z_{0} g(t)+\left[\dot{z}_{0}+\mathrm{i}\left(1+n_{0}\right) z_{0}\right] h(t)\right\} \mathrm{e}^{-\mathrm{i} t},
$$

where $g(t)$ and $h(t)$ are already defined in eq. (36). We interpret these ensemble averages as conditional averages for a fixed set of initial values $z_{0}, \dot{z}_{0}$ and $n_{0}$, so that we can perform the averages with respect to the distribution $P\left(\xi_{0}, \theta_{0}, n_{0}, t=0\right)$ at the end.

We first consider $g(t)=\left\langle X_{1} \exp (-\mathrm{i} \theta)\right\rangle$. The time evolution of $g(t)$ can be obtained by solving the set of four coupled equations for $g_{1}(t) \equiv\left\langle X_{1} \exp (-\mathrm{i} \theta)\right\rangle_{1}$, $g_{2}(t) \equiv\left\langle X_{1} \exp (-\mathrm{i} \theta)\right\rangle_{2}, \quad g_{3}(t) \equiv\left\langle\dot{X}_{1} \exp (-\mathrm{i} \theta)\right\rangle_{1}$ and $g_{4}(t) \equiv\left\langle\dot{X}_{1} \exp (-\mathrm{i} \theta)\right\rangle_{2}$. Introducing a column vector $g(t) \equiv \operatorname{col}\left[g_{1}, g_{2}, g_{3}, g_{4}\right]$, and taking appropriate moments of eqs. (40) we obtain

$$
\dot{\boldsymbol{g}}=\mathbf{N} \cdot \boldsymbol{g},
$$

where

$$
\mathbf{N}=\left(\begin{array}{cccc}
-(\lambda+\mathrm{i} \eta) & \lambda & 1 & 0 \\
\lambda & -(\lambda-\mathrm{i} \eta) & 0 & 1 \\
-\beta^{+} & 0 & -(\lambda+\mathrm{i} \eta) & \lambda \\
0 & -\beta^{-} & \lambda & -(\lambda-\mathrm{i} \eta)
\end{array}\right)
$$

The initial conditions are obtained by using $X_{1}(0)=1, \dot{X}_{1}(0)=0, \theta(0)=0$, and eqs. (41) as $g(0)-\operatorname{col}\left[\delta_{n_{0}, \eta}, \delta_{n_{0},-\eta}, 0,0\right]$. Since the coefficient of $g(t)$ in eq. (42) does not contain $n_{0}$, we are allowed to average $g(0)$ with respect to the initial value $n_{0}$ at this stage. Since $n_{0}= \pm \eta$ with equal probability, we find $g(0)=$ $(1 / 2) \operatorname{col}[1,1,0,0]$. Solving eqs. (43) with this initial condition using the Laplace transform and noting $g(t) \equiv g_{1}(t)+g_{2}(t)$ we find

$$
\bar{g}(s)=\Delta g(s) / \Delta(s),
$$

where

$$
\Delta g(s)=s\left[1+(s+2 \lambda)^{2}\right]+2 \eta^{2}[s+\lambda]+\mathrm{i} \eta[s(s+2 \lambda)+\eta(\eta-2)],
$$




$$
\Delta(s)=\left[(s+2 \lambda)^{2}+1\right]\left[s^{2}+1\right]-4 \eta^{2}[1+\mathrm{i}(s+\lambda)]^{2} .
$$

The correlation function $h(t)$ is obtained in a similar way by solving the coupled set of equations for $h_{1}(t)=\left\langle X_{2} \exp (-\mathrm{i} \theta)\right\rangle_{1}, \quad h_{2}(t)=\left\langle X_{2} \exp (-\mathrm{i} \theta)\right\rangle_{2}$, $h_{3}(t) \equiv\left\langle\dot{X}_{2} \exp (-\mathrm{i} \theta)\right\rangle_{1} \quad$ and $\quad h_{4}(t) \equiv\left\langle\dot{X}_{2} \exp (-\mathrm{i} \theta)\right\rangle_{2} . \quad$ The vector $\boldsymbol{h}(t)=$ $\operatorname{col}\left[h_{1}, h_{2}, h_{3}, h_{4}\right]$ statisfies the same equation (43) with the following initial conditions: $\boldsymbol{h}(0)=\operatorname{col}\left[0,0, \delta_{n_{0}, \eta}, \delta_{n_{0},-\eta}\right]$. The latter follows from $X_{2}(0)=0$, $\dot{X}_{2}(0)=1, \theta(0)=0$ and eqs. (41). However, the coefficient of $h(t)$ in eq. (42) contains the initial value $n_{0}$ this time. Since the averages of $n_{0} \delta_{n_{0},+\eta}$ and $n_{0} \delta_{n_{0},-\eta}$ over the initial distribution, i.e. $n_{0}= \pm \eta$ with equal probability, are $+\eta / 2$ and $-\eta / 2$, respectively, we must solve eqs. (43) once with the initial vector $\boldsymbol{h}^{(1)}(0)=$ $(1 / 2) \operatorname{col}[0,0,1,1]$ and once with $\boldsymbol{h}^{(2)}(0)=(1 / 2) \operatorname{col}[0,0,1,-1]$. The results for $h(t)=h_{1}(t)+h_{2}(t)$ in the Laplace domain in these two cases are

$$
\bar{h}^{(1)}(s)=\Delta h^{(1)}(s) / \Delta(s)
$$

and

$$
h^{(2)}(s)=\Delta h^{(2)}(s) / \Delta(s)
$$

where

$$
\begin{aligned}
& \Delta h^{(1)}(s)=(s+2 \lambda)^{2}+1, \\
& \Delta h^{(2)}(s)=-2 \eta[1+\mathrm{i}(s+\lambda)] .
\end{aligned}
$$

Substitution of eqs. (45) and (46) into eq. (42) yields the Laplace transform of the mean position of the particle as

$$
\begin{aligned}
\langle z(s)\rangle= & z_{0}\left[\Delta g(s+\mathrm{i})+\mathrm{i} \Delta h^{(1)}(s+\mathrm{i})+\mathrm{i} \eta \Delta h^{(2)}(s+\mathrm{i})\right] / \Delta(s+\mathrm{i}) \\
& +\dot{z}_{0} \Delta h^{(1)}(s+\mathrm{i}) / \Delta(s+\mathrm{i})
\end{aligned}
$$

If this result is averaged over an isotropic initial velocity distribution, the second term vanishes. In this case, the mean position of the particle always remains at the origin if it is located there initially.

The stability of the mean position is determined by the characteristic equation $\Delta(s)=0$ where $\Delta(s)$ is given in eq. (45b). We investigated the stability using the Nyquist criterion ${ }^{8}$ ) and found that the mean is stable when

$$
\lambda^{2} \leqslant \lambda_{\mathrm{c}}^{2}\left(\eta^{2}\right)=\left\{4 \eta^{2}\left(5+2 \eta^{2}\right)-1-\left(1+8 \eta^{2}\right)^{3 / 2}\right\} /\left\{4\left[1-2 \eta^{2}+\left(1+8 \eta^{2}\right)^{1 / 2}\right]\right\}
$$


holds and also when $\eta^{2}>3$. The region of stability is depicted in fig. 1. The boundary curve $\lambda^{2}=\lambda_{c}^{2}\left(\eta^{2}\right)$ corresponds to the upper limit of eq. (48). It is observed that the mean position returns to the origin for values of $\lambda^{2}$ and $\eta^{2}$ in the stable region which starts at $\eta^{2}=1$. The emergence of a stability region when $\eta>1$ is due to the fact that the magnetic field starts reversing its direction part of the time when $\eta>1$, and consequently the particle tends to reverse its direction of motion. When $\eta \gg 1$, the magnetic field becomes increasingly more symmetric about $B=0$, and the motion in the reversed direction cancels the motion in the positive direction on the average.

We have investigated the time-dependence of the mean position assuming that the particle is initially located at the origin, i.e. $z_{0}=0$. Then eq. (47) reduces to

$$
\langle z(s)\rangle=\dot{z}_{0} \Delta h^{(1)}(s+\mathrm{i}) / \Delta(s+\mathrm{i})
$$

or in the time domain

$$
\langle z(t)\rangle=\dot{z}_{0} h^{(1)}(t) \mathrm{e}^{-\mathrm{i} t}
$$

where $h^{(1)}(t)$ can be obtained directly by solving the matrix equation $\dot{\boldsymbol{h}}=\mathbf{N} \cdot \boldsymbol{h}$ with the initial condition $h(0)=(1 / 2) \operatorname{col}[0,0,1,1]$ and using $h^{(1)}(t)=$ $h_{1}(t)+h_{2}(t)$, as explained earlier. Fig. 2 shows the magnitude of the mean position, $|\langle z(t)\rangle|$, which is the distance of the mean position from the origin, as

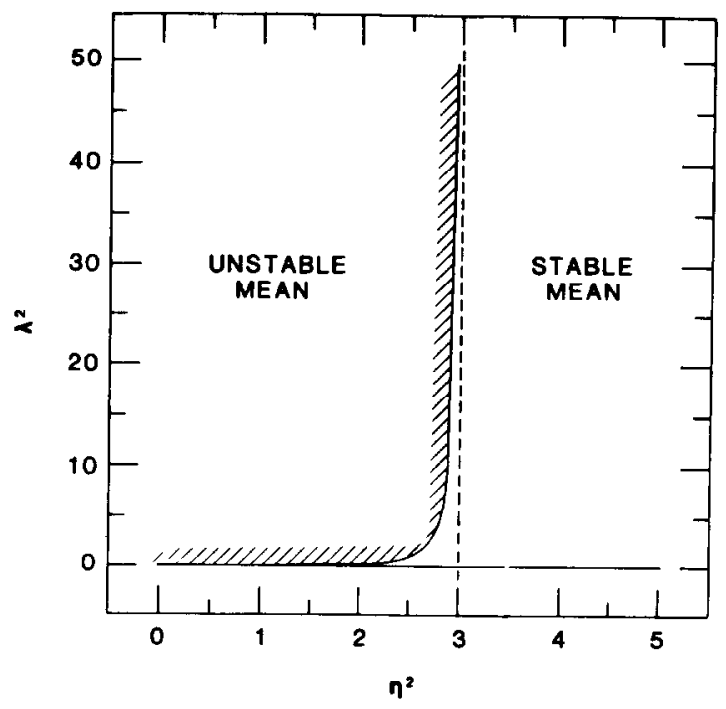

Fig. 1. Regions of stability for the mean as given by eq. (48). 


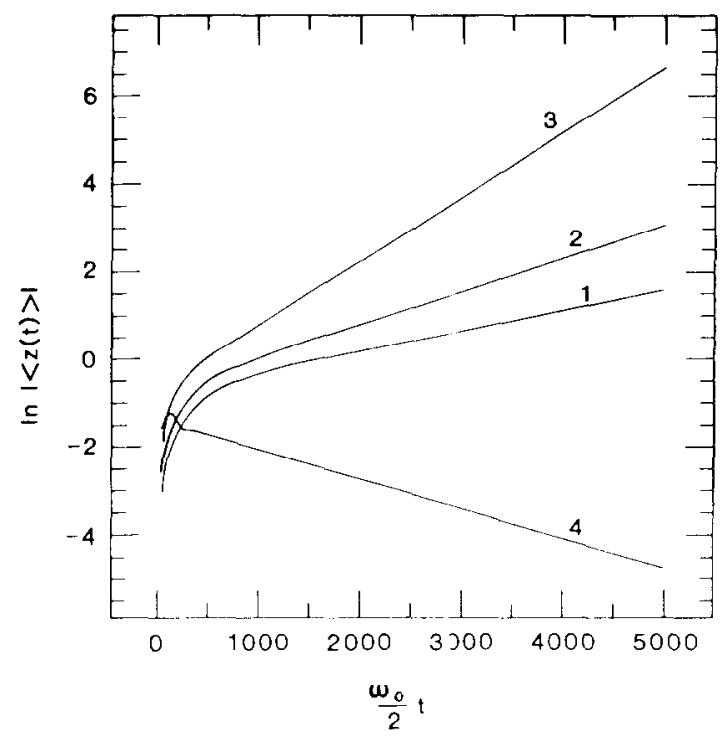

Fig. 2. Behavior of the magnitude of the mean distance $|\langle z(t)\rangle|$ as a function of the rescaled time $\omega_{0} t / 2$ on a logarithmic scale for different sets of parameters: (1) $\eta=0.1, \lambda=10,(2) \eta=0.1, \lambda=2 \pi$, (3) $\eta=0.1, \lambda=3$, and (4) $\eta=\sqrt{3}, \lambda=300$. Cases 1-3 correspond to unstable means whereas case 4 depicts a stable one.

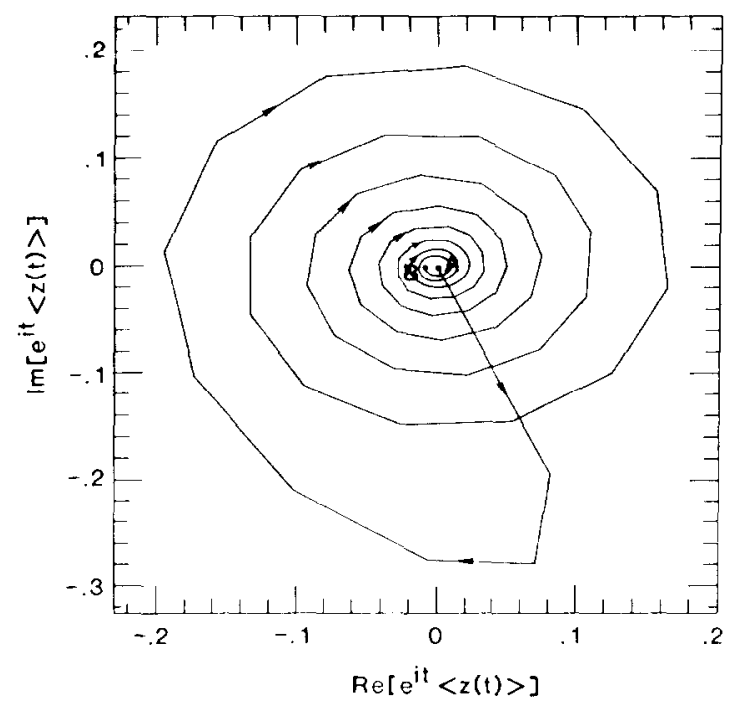

Fig. 3. Case of a stable mean particle trajectory (represented by $\mathrm{e}^{\mathrm{i}}\langle z(t)\rangle$ for convenience) with $\eta=\sqrt{3}$, and $\lambda=300$. Starting at the origin (point A corresponding to $z_{0}=0, \dot{z}_{0}=1$ ) the particle returns to it (point $B$ corresponding to $\omega_{0} t / 2=5000$ ). The trajectory seems jogged because of the long time steps chosen in order to see the return to the origin. 


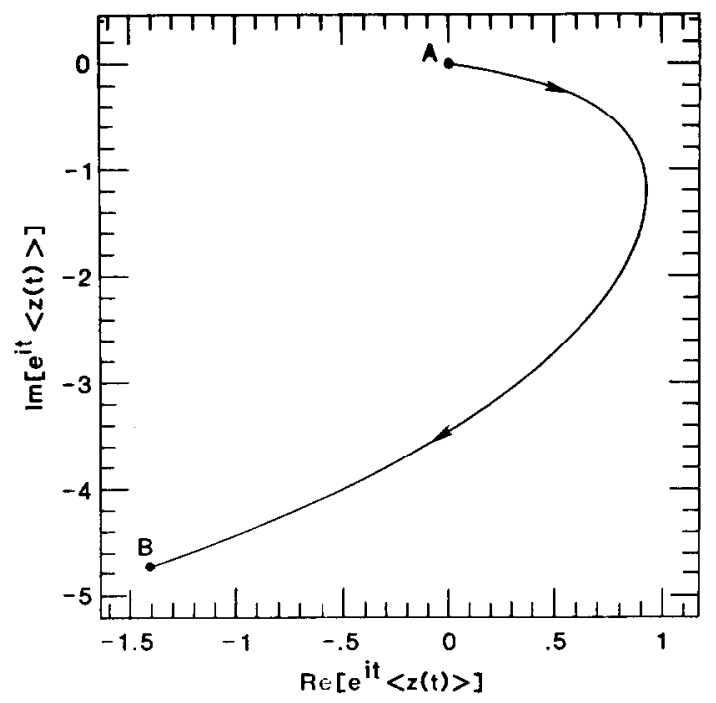

Fig. 4. Same as fig. 3 for an unstable mean particle trajectory with $\eta=0.1$ and $\lambda=10$.

function of the normalized time $\omega_{0} t / 2$ for $\left|\dot{z}_{0}\right|=1$ and for different choices of parameters. When $\eta=0.1$ the mean is unstable, and the distance of the mean position to the origin increases exponentially in time. The case $\eta=\sqrt{3}$ corresponds to a stable mean, for which the particle, starting from the origin at $t=0$, returns exponentially to it on the average.

Figs. 3 and 4 show the mean position of the particle initially located at the origin with a unit velocity in the $x$-direction, i.e. $z_{0}=0$ and $\dot{z}_{0}=1$, for two sets of parameters corresponding to stable and unstable means. For clarity of the figure $\mathrm{e}^{\mathrm{i} t}\langle z(t)\rangle$, rather than $\langle z(t)\rangle$ itself, is plotted on the complex plane.

\section{Variance and standard deviation}

This section is concerned with the calculation of the variance which we define as

$$
\sigma^{2}(t)=\left\langle|z(t)|^{2}\right\rangle-|\langle z(t)\rangle|^{2}
$$

Since the mean position was the object of our concern in the last section, we concentrate here on the mean square distance $\left\langle|z(t)|^{2}\right\rangle$ which is identical to $\left\langle|Z(t)|^{2}\right\rangle$ according to eq. (29). Using eq. (32) we then find

$$
\left\langle|z(t)|^{2}\right\rangle=\left|Z_{0}\right|^{2}\left\langle X_{1}^{2}(t)\right\rangle+2\left\langle X_{1}(t) X_{2}(t)\right\rangle \operatorname{Re}\left(Z_{0}^{*} \dot{Z}_{0}\right)+\left|\dot{Z}_{0}\right|^{2}\left\langle X_{2}^{2}(t)\right\rangle .
$$


For simplicity, here also we assume that the particle is located at the origin initially, so that $Z_{0}=0$ and $\dot{Z}_{0}=\dot{z}_{0}$. Then

$$
\left\langle|z(t)|^{2}\right\rangle=\left|\dot{z}_{0}\right|^{2}\left\langle X_{2}^{2}(t)\right\rangle
$$

To obtain the time evolution of $\left\langle X_{2}^{2}(t)\right\rangle$ we define a column vector $\Gamma(t)=\operatorname{col}\left[\left\langle X_{2}^{2}\right\rangle_{1},\left\langle X_{2}^{2}\right\rangle_{2},\left\langle\dot{X}_{2} X_{2}\right\rangle_{1},\left\langle X_{2} \dot{X}_{2}\right\rangle_{2},\left\langle\dot{X}_{2}^{2}\right\rangle_{1},\left\langle\dot{X}_{2}^{2}\right\rangle\right]$ where the ensemble averages $\langle\ldots\rangle_{1}$ and $\langle\ldots\rangle_{2}$ are over the distributions $P_{1}$ and $P_{2}$, respectively, as before. Using the master equations for $P_{1}$ and $P_{2}$ in eqs. (40), we find the time evolution of $\Gamma(t)$ as

$$
\dot{\Gamma}(t)=\mathbf{S} \cdot \Gamma(t)
$$

where

$$
\mathbf{S}=\left(\begin{array}{rrrrrr}
\lambda & \lambda & 2 & 0 & 0 & 0 \\
\lambda & -\lambda & 0 & 2 & 0 & 0 \\
-\beta^{+} & 0 & -\lambda & \lambda & 1 & 0 \\
0 & -\beta^{-} & \lambda & -\lambda & 1 & 0 \\
0 & 0 & -2 \beta^{+} & 0 & -\lambda & \lambda \\
0 & 0 & 0 & -2 \beta^{-} & \lambda & -\lambda
\end{array}\right)
$$

The initial conditions follow from $X_{2}(0)=0$ and $\dot{X}_{2}(0)=1$ and eqs. (41) as $\Gamma(0)=(1 / 2) \operatorname{col}[0,0,0,0,1,1]$. The function $\left\langle X_{2}^{2}(t)\right\rangle$ is determined from the solution of eqs. (52) as $\left\langle X_{2}^{2}(t)\right\rangle=\left\langle X_{2}^{2}(t)\right\rangle_{1}+\left\langle X_{2}^{2}(t)\right\rangle_{2}$.

We have solved eq. (52) numerically by seeking the eigenvalues $s_{j}$ of the matrix $\mathbf{S}$. The solution is presented as

$$
\boldsymbol{\Gamma}(t)=\sum_{j=1}^{6} \mathrm{e}^{s_{j} t} \mathbf{Q} \cdot \mathbf{d}_{j} \cdot \mathbf{Q}^{-1} \cdot \boldsymbol{\Gamma}(0)
$$

where $\mathbf{Q}$ is the eigenvector matrix, and $\mathbf{d}_{j}$ is a diagonal matrix with all zero diagonal elements except for the $j$ th one. Considering the same sets of parameters that were used to plot the mean distance in fig. 2, we find that the mean square distance always has an eigenvalue with a positive real part which drives it unstable, therefore making the variance $\sigma^{2}(t)$ itself unstable. As shown in fig. 5 , this holds even in the case with $\eta=\sqrt{3}$ and $\lambda=300$ for which the mean distance $|\langle z(t)\rangle|$ is stable. In fig. 6, we plot the mean position $|\langle z(t)\rangle|$ and the standard deviation $\sigma(t)$ together to demonstrate "the mean square instability" (case $\eta=\sqrt{3}$ ) in which the mean is stable (curve 2), but the mean square grows exponentially with time (curve $\left.2^{\prime}\right)$. The second case $(\eta=0.1)$ shown in this 


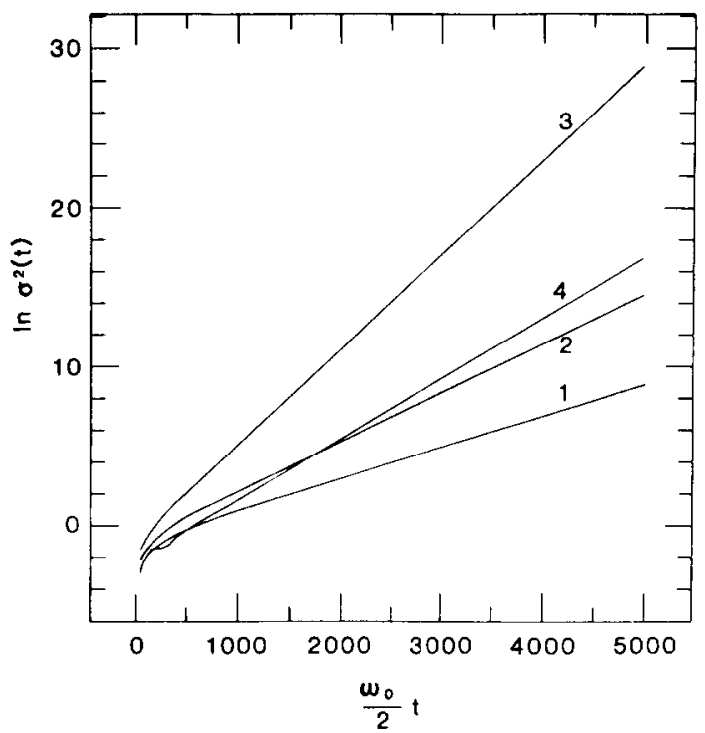

Fig. 5. Behavior of the variance $\sigma^{2}(t)$ vs. $\omega_{0} t / 2$ on a logarithmic scale for the same sets of parameters as in fig. 2, i.e. (1) $\eta=0.1, \lambda=10$, (2) $\eta=0.1, \lambda=2 \pi$, (3) $\eta=0.1, \lambda=3$ and (4) $\eta=\sqrt{3}, \lambda=300$.

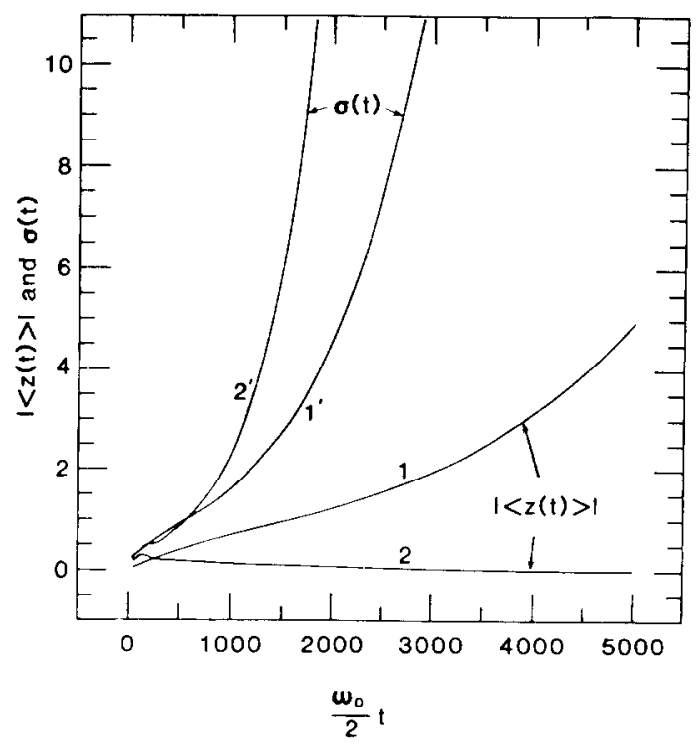

Fig. 6. Variation of the mean distance $|\langle z(t)\rangle|$ and standard deviation $\sigma(t)$ vs. $\omega_{0} t / 2$ for: (1) and $\left(1^{\prime}\right)$ $\eta-0.1, \lambda=10,(2)$ and $\left(2^{\prime}\right) \eta=\sqrt{3}, \lambda=300$. 


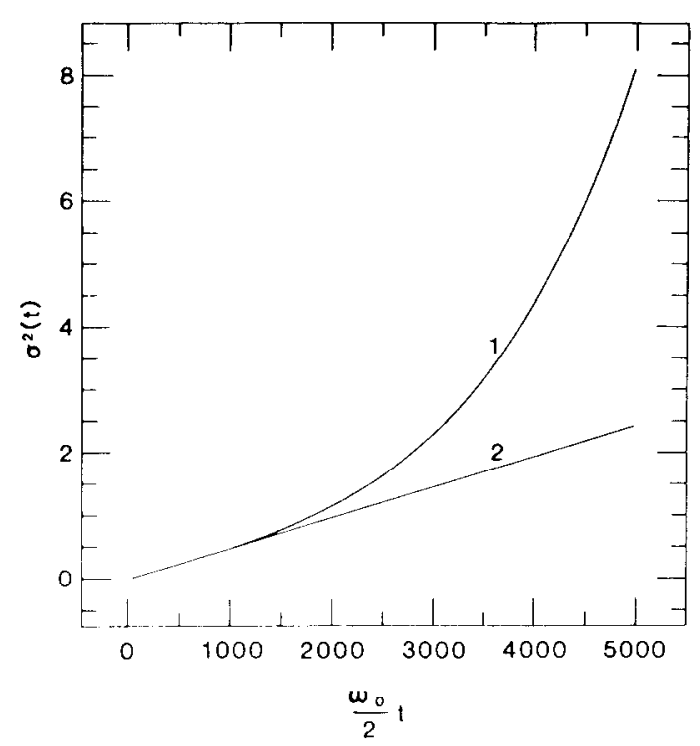

Fig. 7. Variation of the variance $\sigma^{2}(t)$ vs. $\omega_{0} t / 2$ with (curve 1) and without (curve 2) the induced electric field for $\eta=0.1$ and $\lambda=30$.

figure corresponds to the "mean instability" in which the mean (curve 1) itself is unstable. It is noted that although the mean square distance $\left\langle|z(t)|^{2}\right\rangle$ (not plotted here) displays an oscillatory increase, the variance $\sigma^{2}(t)$ which is the mean square deviation from the mean distance increases smoothly and shows no oscillations. Fig. 7 shows the initial linear increase of $\sigma^{2}(t)$ corresponding to the diffusion region ( $\boldsymbol{B}$ field only) and then gradually the exponential behavior takes over when the induced $\boldsymbol{E}$ field term in eq. (1) becomes dominant. Since we know that for this case $(\eta=0.1)$ the mean is unstable, we can conclude that the particle is quickly lost from the central region where it started. Note that in the stable mean case $(\eta=\sqrt{3})$ the particle has wild variations in its trajectory, but it still remains in the central region on the average.

\section{Summary}

The motion of a charged particle in a randomly varying uniform magnetic field has been investigated. A stability analysis showed that the mean position is unstable unless magnetic field fluctuations are large enough compared to the uniform background. When the mean position is unstable, the particle quickly leaves the central region where it starts. When the mean is stable, even though the particle, on the average, remains in the central region, it can acquire wild 
oscillations in its trajectory (unstable mean square). Since a real system of charged particles such as a plasma has a large number of charged particles, it would be interesting to see how collisions would affect our present conclusion.

\section{Acknowledgements}

The authors acknowledge many fruitful discussions with Dr. M.E. Fenstermacher. This work was supported by an NSF grant.

\section{Appendix}

We consider the following stochastic differential equation:

$$
\dot{X}(t)=g(X, \varepsilon(t))+h(X, \varepsilon(t)) \cdot f(t),
$$

where $\{f(t)\}$ is a Gaussian process with a zero mean and a correlation function

$$
\left\langle f(t) f^{\mathrm{T}}\left(t^{\prime}\right)\right\rangle=2 \mathrm{~B} \delta\left(t-t^{\prime}\right) .
$$

The process $\{\varepsilon(t)\}$ is assumed to be a Markov process characterized by a transition probability per unit time $W\left(\varepsilon^{\prime} \mid \varepsilon, t\right)$ at time $t$, from $\varepsilon$ to $\varepsilon^{\prime}$. The total transition probability is $\Gamma(\varepsilon, t)=\int \mathrm{d} \varepsilon^{\prime} W\left(\varepsilon^{\prime} \mid \varepsilon, t\right)$. We want to obtain the Fokker-Planck equation for the probability $P(X, \varepsilon, t)$ of finding $X(t)$ and $\varepsilon(t)$ at $\boldsymbol{X}$ and $\varepsilon$ at time $t$. The important observation that enables the solution of this problem is that the joint process $\{\boldsymbol{X}(t), \boldsymbol{\varepsilon}(t)\}$ is a Markov process, because the knowledge of $\boldsymbol{X}\left(t_{0}\right)$ and $\varepsilon\left(t_{0}\right)$ completely determines the future values of $\boldsymbol{X}(t)$ and $\varepsilon(t)$ for $t \geqslant t_{0}$.

We start from the Chapman-Kolmogorov equation

$$
P(\boldsymbol{X}, \boldsymbol{\varepsilon}, t+\Delta t)=\int \mathrm{d} \boldsymbol{X}^{\prime} \mathrm{d} \varepsilon^{\prime} P\left(\boldsymbol{X}, \varepsilon, t+\Delta t \mid \boldsymbol{X}^{\prime}, \boldsymbol{\varepsilon}^{\prime}, t\right) P\left(\boldsymbol{X}^{\prime}, \boldsymbol{\varepsilon}^{\prime}, t\right) .
$$

The crucial step is

$$
\begin{aligned}
P\left(\boldsymbol{X}, \boldsymbol{\varepsilon}, t+\Delta t \mid \boldsymbol{X}^{\prime}, \varepsilon^{\prime}, t\right)= & \delta\left(\varepsilon-\varepsilon^{\prime}\right)[1-\Delta t \Gamma(\varepsilon, t)] P_{\varepsilon}\left(\boldsymbol{X}, t+\Delta t \mid \boldsymbol{X}^{\prime}, t\right) \\
& +\Delta t W\left(\varepsilon \mid \varepsilon^{\prime}, t\right) \delta\left(\boldsymbol{X}-\boldsymbol{X}^{\prime}\right),
\end{aligned}
$$

where the first term on the right-hand side represents the case in which there is no transition during $\Delta t$ from $\varepsilon^{\prime}$ to $\varepsilon$, and $X(t)$ changes from $X^{\prime}$ to $X$ according 
to eq. (A.1) with a given $\varepsilon$. The second term accounts for transitions from $\varepsilon^{\prime}$ to $\boldsymbol{E}$ in $\Delta t$ in which $\boldsymbol{X}(t)$ does not change appreciably by diffusional processes. Here lies an important physical restriction on the nature of the $\{\varepsilon(t)\}$ process: it is implied that $\varepsilon(t)$ evolves in time by making sudden jumps and is constant between the jumps. On the other hand, $\boldsymbol{X}(t)$ evolves more slowly in time by diffusion, according to eq. (A.1).

The function $P_{\varepsilon}\left(\boldsymbol{X}, t+\Delta t \mid \boldsymbol{X}^{\prime}, t\right)$ is the probability of finding $\boldsymbol{X}(t+\Delta t)$ at $\boldsymbol{X}$ knowing that $\boldsymbol{X}(t)=\boldsymbol{X}^{\prime}$ for a fixed value of $\varepsilon(t) \equiv \varepsilon$. It can be expanded as

$$
P_{\varepsilon}\left(\boldsymbol{X}, t+\Delta t \mid \boldsymbol{X}^{\prime}, t\right)=\delta\left(\boldsymbol{X}-\boldsymbol{X}^{\prime}\right)+\Delta t \partial P_{\varepsilon}\left(\boldsymbol{X}, t^{\prime} \mid \boldsymbol{X}^{\prime}, t\right) /\left.\partial t^{\prime}\right|_{t^{\prime} \rightarrow t}
$$

where we have used

$$
\left.\lim _{t^{\prime} \rightarrow t} P_{\varepsilon}\left(\boldsymbol{X}, t^{\prime}\right) \mid \boldsymbol{X}, t\right)=\delta\left(\boldsymbol{X}-\boldsymbol{X}^{\prime}\right)
$$

The conditional distribution function $P_{\varepsilon}\left(\boldsymbol{X}, t^{\prime} \mid \boldsymbol{X}^{\prime}, t\right)$ satisfies the following Fokker-Planck equation:

$$
\begin{aligned}
\partial P_{\varepsilon}\left(\boldsymbol{X}, t^{\prime} \mid \boldsymbol{X}^{\prime}, t\right) / \partial t^{\prime}= & -\left(\partial / \partial X_{j}\right)\left[\eta_{j}(\boldsymbol{X}, \varepsilon) P_{\varepsilon}\right] \\
& +(1 / 2)\left(\partial^{2} / \partial X_{j} \partial X_{k}\right)\left[\sigma_{j k}(\boldsymbol{X}, \varepsilon) P_{\varepsilon}\right]
\end{aligned}
$$

The expressions of $\eta_{j}$ and $\sigma_{j k}$ will be given below. The substitution of eqs. (A.4)-(A.7) in (A.3) yields

$$
\begin{aligned}
\partial P(\boldsymbol{X}, \boldsymbol{\varepsilon}, t) / \partial t= & -\left(\partial / \partial X_{j}\right)\left[\eta_{j}(\boldsymbol{X}, \boldsymbol{\varepsilon}) P(\boldsymbol{X}, \boldsymbol{\varepsilon}, t)\right] \\
& +(1 / 2)\left(\partial^{2} / \partial X_{j} \partial X_{k}\right)\left[\sigma_{j k}(\boldsymbol{X}, \boldsymbol{\varepsilon}) P(\boldsymbol{X}, \boldsymbol{\varepsilon}, t)\right] \\
& +\int \mathrm{d} \boldsymbol{\varepsilon}^{\prime}\left[W\left(\varepsilon \mid \varepsilon^{\prime}, t\right) P\left(\boldsymbol{X}, \boldsymbol{\varepsilon}^{\prime}, t\right)-W\left(\boldsymbol{\varepsilon}^{\prime} \mid \boldsymbol{\varepsilon}, t\right) P(\boldsymbol{X}, \boldsymbol{\varepsilon}, t)\right],
\end{aligned}
$$

where

$$
\eta_{j}(\boldsymbol{X}, \varepsilon)=\lim _{\Delta t \rightarrow 0}\left\langle\Delta X_{j}(t) / \Delta t\right\rangle
$$

and

$$
\sigma_{j k}(X, \varepsilon)=\lim _{\Delta t \rightarrow 0}\left\langle\Delta X_{j}(t) \Delta X_{k}(t) / \Delta t\right\rangle
$$

and can be calculated from the basic equation governing diffusion. When the 
latter has the form given in (eq. A.1) we find

$$
\eta_{j}(\boldsymbol{X}, \varepsilon)=g_{j}+\left(\partial h_{j l} / \partial X_{m}\right) h_{m n} B_{l n}
$$

and

$$
\sigma_{j k}(X, \varepsilon)=2 h_{j l} B_{l m} .
$$

Eqs. (A.8), (A.9) and (A.10) constitute the basic equations to study the jump and diffusion processes.

\section{References}

1) S. Chandrasekhar, Plasma Physics (Univ. of Chicago Press, Chicago, 1960).

2) N.G. Van Kampen, J. Stat. Phys. 25 (1981) 431.

3) N.G. Van Kampen, Stochastic Processes in Physics and Chemistry (North-Holland, Amsterdam, 1981).

4) R.C. Bourret, U. Frisch and A. Pouquet, Physica 65 (1973) 303.

5) N.G. Van Kampen, Phys. Rep. 24C (1976) 171.

6) J. McKenna and J.A. Morisson, J. Math. Phys. 11 (1970) 2348.

7) U. Frisch, in: Probabilistic Methods in Applied Mathematics, A.T. Bharucha-Reid, ed. (Academic Press, New York, 1968).

8) J.J. DiStefano, A.R. Stubberud and I.J. Williams, Schaum Outline of Feedback and Control Systems (McGraw-Hill, New York, 1967). 